\title{
Presencia de hifas de cándida en adultos con mucosa oral clínicamente saludable
}

Salazar M, Sacsaquispe S. J. Presencia de hifas de candida en adultos con mucosa oral clínicamente saludable. Rev Estomatol Herediana 2005; 15(1): 54 - 59

\section{RESUMEN}

El objetivo del presente estudio fue determinar la presencia de hifas y/o pseudohifas de cándida en sujetos adultos con mucosa oral clínicamente saludable. Se examinaron 120 sujetos entre 20 y 59 años de edad. Se efectuaron frotices de mucosa oral para tinción PAS y cultivo en agar Sabouraud. Para el análisis se utilizó la prueba chi-cuadrado para determinar su relación con edad, sexo, uso de prótesis, xerostomía, uso de medicamentos, índice de higiene oral, especie de cándida y cantidad de UFC/ml. Del total de sujetos, 6 (5\%) de ellos presentaron hifas y/o pseudohifas de cándida, 5 de estos presentaron $<400 \mathrm{UFC} / \mathrm{ml}$. No se encontró relación entre la presencia de cándida con respecto a la edad, género, uso de medicamentos, índice de higiene oral, especie de cándida, mientras que si se encontró relación entre la presencia de hifas y/o pseudohifas de cándida y el uso de prótesis dental, xerostomía y UFC/ml, hallándose relación estadística altamente significativa $(\mathrm{p}<0.01)$. En conclusión, puede existir la presencia de hifas y/o pseudohifas de cándida en sujetos adultos con mucosa oral clínicamente saludable sin evidencia clínica de infección candidiásica.

Palabras clave: CANDIDIASIS / CULTIVO / XEROSTOMÍA / PRÓTESIS.

\section{Candida hyphas presence in adults with clinically healthy oral mucous} ABSTRACT

The purpose of this study was to determine the presence of Candida hyphas and/or pseudohyphas in adults with clinically healthy oral mucous. 120 subjects were examined, their age ranged between 20 and 59 years. Oral mucous swabs were made for PAS smear and culture in Sabouraud Agar. The Chi-square test was used to analyse and determine its relationship with age, sex, denture use, xerostomy, medications use, oral hygiene index, Candid species and amount of UFC/ml. From the total, 6 (5\%) of them presented Candida hyphas and/or pseudohyphas; 5 presented $<400 \mathrm{UFC} / \mathrm{ml}$. A relationship among Candida hyphas and/or pseudohyphas presence with regard to age, gender, medications use, oral hygiene index, Candid species was not found. However, a relationship among Candida hyphas and/or pseudohyphas presence and denture use, xerostomy and amount of $\mathrm{UFC} / \mathrm{ml}$ was found, with a highly significant statistical relationship $(\mathrm{p}<0.01)$. In conclusion, it may exist presence of Candida hyphas and/or pseudohyphas in adults with clinically healthy oral mucous without evidence of candidiasis infection.

Keywords: CANDIDIASIS / CULTURE / XEROSTOMY / DENTURE.
Mariela Salazar Vasquez ${ }^{1}$ Sonia Sacsaquispe Contreras ${ }^{2}$

${ }^{1}$ Cirujano - Dentista. Alumna del Residentado en Periodoncia.

2Docente del Departamento de Medicina, Cirugía y Patología Oral. Facultad de Estomatología. Universidad Peruana Cayetano Heredia.

\section{Correspondencia}

Sonia Sacsaquispe Contreras,

Av. Honorio Delgado 430, Lima 31 - Perú. Telf.: 3811950.

E-mail: soniasc@upch.edu.pe

Aceptado para publicación : 29 de junio del 2005

\section{Introducción}

La cándida es un organismo comensal y forma parte de la flora oral normal del ser humano, se encuentra entre el $30 \%$ y $70 \%$ de la población, y es capaz de producir infecciones ocasionales dentro de la cavidad bucal cuando se presentan los factores apropiados que predisponen a ello (1-6), su transformación en patógeno se relaciona con factores locales o sistémicos muy difíciles de reproducir en el campo experimental. El porcentaje de portadores de Candida albicans varía grandemente y la mayoría de investigadores están de acuerdo que la levadura se encuentra comúnmente en la boca de personas saludables y que es encontrada en un porcentaje significativo $(5,6)$. La tasa promedio de portadores en personas saludables y pacientes es de $18 \%$ y $41 \%$ respectivamente (5).

La C. albicans suele presentarse como una célula oval levaduriforme de 2 a $4 \mu \mathrm{m}$ con paredes finas; en tejidos infectados se ha identificado formas filamentosas (hifas) que son estructuras microscópicas tubulares de 3 a $4 \mu \mathrm{m}$ de diámetro, que contienen múltiples unidades celulares divididas por septos y pueden surgir a partir de blastosporas o de hifas existentes; y pseudohifas que corresponden a células alargadas de levadura que se producen cuando las yemas continúan su crecimiento y que permanecen unidas entre sí (6).

La infección por cándida es denominada candidiasis o candidosis y puede cursar en forma aguda o crónica dando lugar a infecciones superficiales o profundas. Existe concordancia entre la mayoría de los investigadores con respecto a que las hifas están asociadas con su capacidad invasiva $(4,6)$. Esta infección se presenta en personas de 
edad variable aunque se observa con mayor frecuencia en lactantes, ancianos y personas con factores predisponentes generales y locales (2). La candidiasis puede servir de indicador clínico que señala la presencia de una enfermedad significativa subyacente $(3,5)$.

Es la micosis más común en la cavidad bucal humana (6), una infección oportunista causada por un hongo saprofítico del género cándida, el cual incluye ocho especies de hongos, la más común de las cuales es la C. albicans $(7,8)$. Es la especie que mas comúnmente se aísla en esta enfermedad, apareciendo en más del 70\% de los aislamientos $(4,6)$. En portadores de cándida se encuentra más frecuentemente en la lengua, mejilla y paladar, sitios que coinciden con las regiones más densamente colonizadas (4).

La línea divisoria entre la colonización candidiásica y la infección es bastante confusa aunque se ha sugerido que ninguna forma de candidiasis superficial ni sistémica puede iniciarse en ausencia de patología subyacente (9). Parece ser que la sola presencia del hongo no es suficiente para producir la enfermedad. En consecuencia, debe existir una penetración de los tejidos, aunque tal invasión por lo regular es superficial y solo se presenta en ciertas circunstancias.

La transformación de comensal en patógeno puede estar asociada a la virulencia del microorganismo; sin embargo a diferencia de otras enfermedades en la candidiasis bucal, se acepta que, los factores del huésped, son de una importancia critica en la patogénesis de la infección, igual o mayor, que la propia virulencia del hongo. En suma, los factores locales ambientales, la virulencia microbiana y determinadas características del huésped actúan como determinantes de la susceptibilidad a la enfermedad $(3,4,9)$.

Las condiciones que pueden predisponer a un paciente a desarrollar candidiasis oral se pueden clasificar en: a) Factores que alteran el estado inmune del huésped como Discrasias sanguíneas o enfermedades malignas avanzadas, en ancianos e infantes, terapia con radiación y quimioterapia, infección por HIV u otros desórdenes de inmunodeficiencia, anomalías endocrinas, Diabetes mellitus, Hipotiroidismo o hipoparatiroidismo, embarazo, terapia con corticoesteroides, Hipoadrenalismo) y b) Factores que alteran la mucosa oral como antibióticoterapia, mala higiene oral o de la prótesis, malnutrición, mala absorción gastrointestinal, deficiencias de hierro, ácido fólico o vitaminas, saliva ácida, dieta rica en carbohidratos, fumadores empedernidos, displasia epitelial oral, xerostomia $(3,4,6,9)$.

Los estudios en relación con hifas de cándida como el deArendorf y Walker (10) en 54 sujetos con edades comprendidas entre los 17 y 61 años encontraron en tres $(5,56 \%)$ sujetos adultos saludables sin historia de candidiasis oral; Rindum (1) refiere haber encontrado hifas y/o pseudohifas de cándida en sujetos saludables con mucosa oral sana del 4 al $47 \%$; pero; si se considera solo a los no fumadores, del 4 al $24 \%$.

El propósito del presente estudio fue determinar mediante exámenes citológicos la presencia de hifas y/o pseudohifas de cándida y mediante cultivos la presencia de unidades formadoras de colonias en sujetos adultos con mucosa oral clínicamente saludable y relacionar los resultados con factores predisponentes.

\section{Materiales y método}

Se examinaron 120 sujetos adultos entre 20 y 59 años de edad que acudieron al Servicio de Diagnóstico de la Clínica Estomatológica de la Universidad Peruana Cayetano Heredia, y que aceptaron voluntariamente participar en el estudio firmando una hoja de consentimiento.

En este estudio se definió mucosa oral clínicamente saludable como aquella que conserva las características propias de cada región (revestimiento, especializada, masticatoria) con buena humedad y en ausencia de lesiones primarias y/o secundarias.

\section{Criterios de exclusión}

Se consideraron los siguientes criterios de exclusión: presencia de discrasia sanguínea, enfermedades malignas avanzadas, infección por VIH u otros desordenes de inmunodeficiencia, Sín- drome de Sjögren, alteraciones endocrinas, diabetes, hipotiroidismo o hipoparatiroidismo, hipoadrenalismo, leucoplasia, terapia con radiación y quimioterapia, con corticoesteroides, antimicóticos, antibióticoterapia, malnutrición, mala absorción gastrointestinal, personas embarazadas, fumadores, personas con incapacitadad física o mental que influenciaban a mantener buena higiene oral.

\section{Criterios de inclusión}

Sujetos adultos de 20 a 59 años de edad de ambos sexos, que al examen clínico no presenten manifestaciones de candidiasis oral en cualquiera de sus formas (pseudomembranosa, eritematosa, hiperplásica y queilitis comisural).

\section{Variables}

Hifas y/o pseudohifas de cándida. Se determinó tomando frotices de mucosa oral a los cuales se les tiñó con PAS (periodic acid-Schiff). Se registró como positivo cuando al observar al microscopio óptico se encuentran estructuras filamentosas teñidas de color rojo a púrpura, que pueden estar tabicadas o no.

- Portadores de prótesis dental removible total o parcial.

- Xerostomía : determinada por la "prueba de la paleta”.

- Higiene oral: Se determinó de acuerdo al índice de Greene-Vermillon como bueno, regular o malo.

- Uso de medicamentos : Se agruparon en : antidepresivos, antihistamínicos, anticolinérgicos, antiparkinsonianos, antipsicóticos, diuréticos, sedativos, antihipertensivos, simpático miméticos.

- Especie de cándida: Se realizo la prueba del tubo germinativo para el diagnóstico, es así que la presencia de $C$. albicans se determinó mediante la prueba del tubo germinativo positivo y a la ausencia de tubo germinativo se consideró Candida spp.

- Recuento de unidades formadoras de colonia: Se observó el crecimiento de las colonias de cándida en medio agar Sabouraud, y luego se realizó el recuento de unidades formadoras de colonia de cándida (UFC/ml.). 


\section{Técnica y procedimiento}

1. Fase Clínica.

Se realizaron frotices de las zonas de lengua, paladar duro y carrillo con un hisopo estéril (Fig. 1); el que se extendió en una lámina portaobjetos, se rotularon y colocaron en alcohol isopropílico al 95\% para su respectiva fijación y posterior tinción en coloración PAS (Fig. 2). Con otro hisopo estéril se procedió a realizar otros frotices y se colocaron en un tubo de ensayo (Fig. 3), completamente hermético y estéril, conteniendo éste 1,8 $\mathrm{ml}$ de agua destilada como vehículo para su posterior cultivo.

2. Fase de laboratorio

a) Histopatológico : Las muestras fueron teñidas con el reactivo Peryódico Ácido Schiff (PAS) en el Laboratorio de Patología Oral de la Facultad de Estomatología de la Universidad Peruana Cayetano Heredia. Luego se observaron al microscopio óptico con aumentos de $10 \mathrm{X}$ y $40 \mathrm{X}$. Los resultados se anotaron en la ficha como positivo (+) cuando estaban presentes las hifas y/o pseudohifas de cándida y negativo (-) en ausencia.

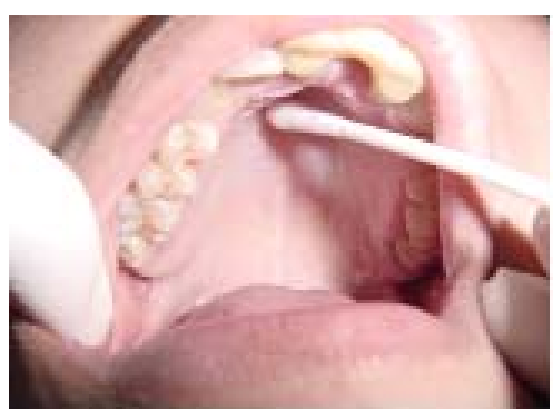

Figura 1. Frotis de paladar.

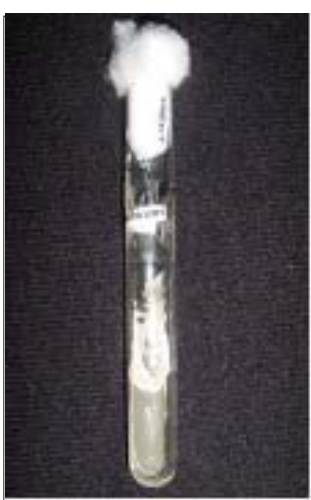

Figura 4. Candida albicans, tubo germinativo positivo. b) Cultivo : Las muestras recogidas en los tubos de ensayo fueron procesadas en el Laboratorio de Micología del Instituto de Medicina Tropical “Alexander Von Humboldt” de la Universidad Peruana Cayetano Heredia, para ser sembradas en placas petri con agar Sabouraud y luego incubadas a $35^{\circ} \mathrm{C}$ por 48 horas. Previamente, para confirmar la presencia de hifas y/o pseudohifas y determinar la cantidad de muestra a cultivarse se realizó la prueba de $\mathrm{KOH}$. Se definió como positivo (+) cuando hubo presencia de levaduras, blastosporas, hifas y/o pseudohifas. Se determinó la especie de cándida mediante la prueba del tubo germinativo : tubo germinativo positivo es C. albicans (Fig. 4) y en ausencia se consideró Candida spp. También se observó el crecimiento de las colonias de la cándida en medio agar Sabouraud, como formaciones redondeadas de color crema, consistencia pastosa y textura lisa o rugosa (Fig. 5) y luego se realizó el recuento de UFC, contabilizándose cada una de ellas.

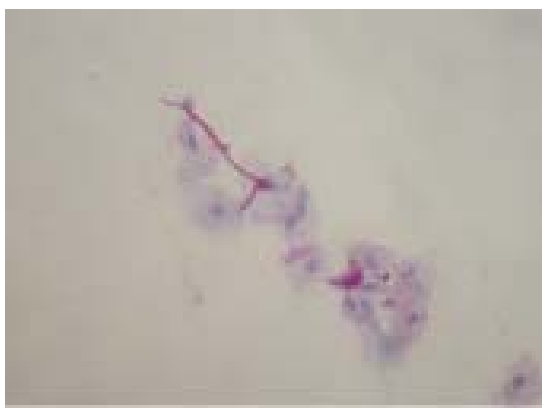

Figura 2. Citología mostrando hifas de cándida (PAS, 200X).

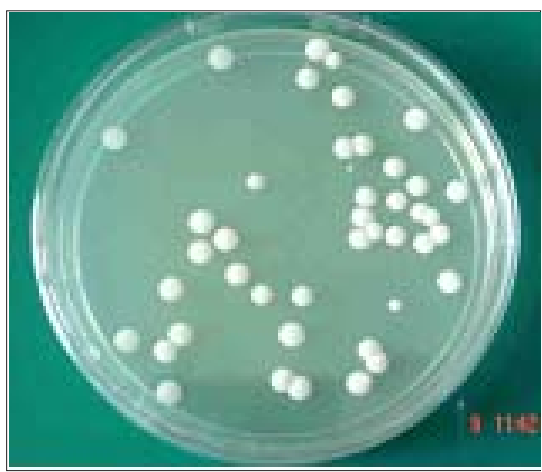

Figura 5. Cultivo mostrando colonias de cándida (Agar de Saboraud).

\section{Análisis etadístico}

Se realizó análisis univariado y bivariado para determinar la asociación entre las variables empleando la prueba chi-cuadrado. Mediante el análisis de regresión logística se evaluó la influencia de cada variable con respecto al desarrollo de hifas y/o pseudohifas de cándida, a un nivel de significancia del 95\%. Se realizaron además curvas de características operativas para el receptor (ROC), para determinar un punto de corte para el recuento de colonias para determinar la sensibilidad y especificidad.

\section{Resultados}

Se examinaron 120 sujetos cuyas edades estuvieron comprendidas entre 20 y 59 años, el promedio de las edades fue de 37,6 (SD=11,1). La mayor frecuencia $(30 \%)$ se encontró para el grupo etario de 20 a 29 años de edad, el 43,33\% fueron varones y 56,7\% mujeres. De ellos, se encontró que seis (5\%) sujetos desarrollaron hifas y/o pseudohifas de cándida, mientras que 114 (95\%) no desarrollaron ( $\mathrm{p}>0,05)$.

De los sujetos estudiados se encontró que 22 (18,33\%) usaban prótesis dental (Fig. 6) y 98 (81,67\%) no usaban,

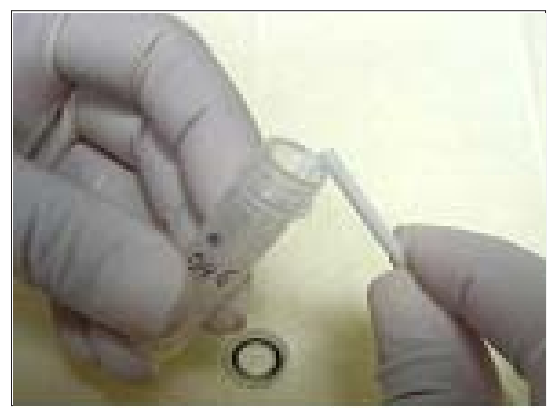

Figura 3. Colocación del hisopo en tubo de ensayo para cultivo.

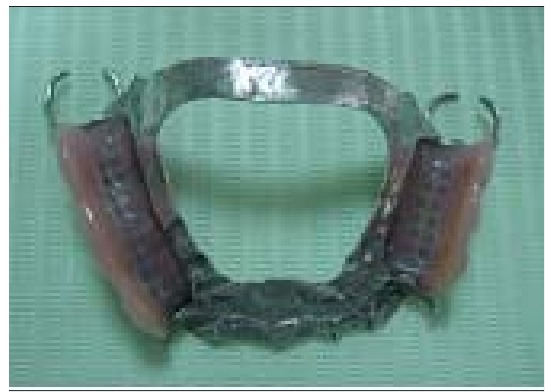

Figura 6. Prótesis dental removible. 
Tabla 1. Resultados del examen directo en coloración PAS según el uso de prótesis

\begin{tabular}{lllllll}
\hline & \multicolumn{2}{l}{ PAS $(+)$} & \multicolumn{2}{c}{ PAS $(-)$} & \multicolumn{2}{c}{ Total } \\
Prótesis & $\mathrm{n}$ & $\%$ & $\mathrm{n}$ & $\%$ & $\mathrm{n}$ & $\%$ \\
\hline $\mathrm{Si}$ & 4 & $3,33(66,66)$ & 18 & $15(15,79)$ & 22 & 18.33 \\
No & 2 & $1,67(33,33)$ & 96 & $80(84,21)$ & 98 & 81.67 \\
Total & 6 & $5,00(100)$ & 114 & $95(100)$ & 120 & 100 \\
\hline
\end{tabular}

Chi cuadrado $=9,85 \mathrm{p}=0,002$

Tabla 2. Resultados del examen directo en coloración PAS según la presencia de xerostomía

\begin{tabular}{lllllll}
\hline & \multicolumn{2}{l}{ PAS $(+)$} & \multicolumn{2}{c}{ PAS $(-)$} & \multicolumn{2}{c}{ TOTAL } \\
Xerostomía & $\mathrm{n}$ & $\%$ & $\mathrm{n}$ & $\%$ & $\mathrm{n}$ & $\%$ \\
\hline SI & 5 & $4,17(83,33)$ & 21 & $17,50(18,42)$ & 26 & 21,67 \\
NO & 1 & $0,83(16,66)$ & 93 & $77,50(81,58)$ & 94 & 78,33 \\
Total & 6 & $5,00(100)$ & 114 & $95,00(100)$ & 120 & 100 \\
\hline
\end{tabular}

Chi cuadrado $=14,15 \mathrm{p}=0,0002$

Tabla 3. Distribución de los sujetos con resultados positivos al examen directo en coloración PAS según, edad, sexo, uso de prótesis, xerostomía, condición de higiene oral, uso de medicamentos, especie de cándida y cantidad de UFC.

\begin{tabular}{llllllll}
\hline casos & edad & sexo & prótesis & xerostomía & higiene & medicamentos & especie \\
\hline 1 & 38 & F & SI & NO & B & NO & C. albicans \\
2 & 21 & M & NO & SI & R & NO & C. albicans \\
3 & 46 & F & SI & SI & M & NO & C. albicans \\
4 & 56 & F & SI & SI & NA & NO & C. albicans \\
5 & 24 & M & NO & SI & R & NO & C. albicans \\
6 & 55 & F & SI & SI & R & SI & C. albicans \\
\hline
\end{tabular}

UFC: unidades formadoras de colonia, B: buena, R: regular, M: mala, NA: no aplicable.

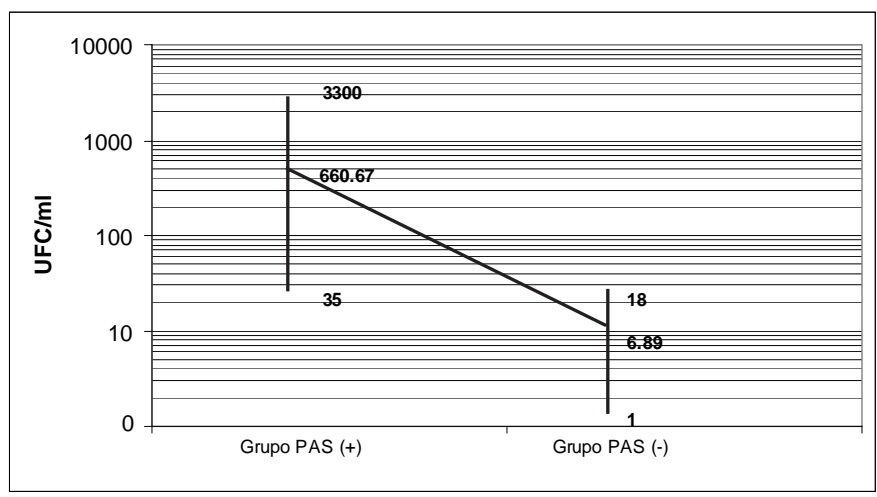

Figura 7. Rangos y promedios de unidades formadoras de colonias de cándida (UFC/ml) en los grupos con PAS (+) y PAS (-).

encontrándose diferencia significativa $(\mathrm{p}<0,005)$ (Tabla 1). Al aplicar regresión logística se encontró influencia del uso de prótesis sobre la presencia de hifas y/o pseudohifas de cándida $(\mathrm{p}=0,048)$, así mismo se encontró que los que usan prótesis tienen una probabilidad de 98,21\% de desarrollarlas.

En los sujetos del grupo de estudio se encontró que $21,67 \%$ presentaron xerostomía y 78,33\% no presentaron xerostomía, existiendo diferencia esta- dística altamente significativa $(\mathrm{p}<0,001)$ (Tabla 2). Al aplicar regresión logística se encontró influencia de la presencia de xerostomía sobre la presencia de hifas y/o pseudohifas de cándida $(\mathrm{p}=0,012)$.

Con respecto a la condición de higiene oral, 28 sujetos (23,33\%) presentaron buena higiene oral, 76 sujetos (63,33\%) regular, 11 sujetos (9,17\%) mala higiene oral y a cinco sujetos $(4,17 \%)$ se les consideró “no aplicable” por presentar menos de dos o ninguna pieza a evaluar ( $p>0,05)$. En relación con el uso de medicamentos, la mayoría de sujetos no consumían; es decir, 106 sujetos (88,33\%), ( $\mathrm{p}>0,05)$. Al determinar la especie de cándida, se encontró que 31 sujetos $(25,83 \%)$ desarrollaron $C$. albicans, dos sujetos $(1,67 \%)$ presentaron Candida spp y 87 sujetos (72,50\%) no presentaron ningún crecimiento de cándida; ( $\mathrm{p}>0,05)$.

En la Tabla 3 se puede observar la distribución de los 6 sujetos que presentaron hifas y/o pseudohifas de cándida, coloración PAS (+) y sus características según edad, género, uso de prótesis, presencia de xerostomía, condición de higiene oral, uso de medicamentos, especie de cándida y cantidad de $\mathrm{UFC} / \mathrm{ml}$. El grupo de "frotis positivo" tuvo un promedio de 660,67 UFC/ml, y el grupo de "frotis negativo" tuvo un promedio de 6,89 UFC/ml. Al evaluar la relación entre el recuento de UFC/ml. y el resultado del frotis en coloración PAS se encontró diferencia altamente significativa $(\mathrm{p}<0,001)$ (Fig. 7). 
Figura 8. Distribución de sujetos con PAS (+) y PAS (-) según el recuento de colonias de cándida (UFC/ml)

En la Figura 8 se muestra la distribución de ambos grupos según el recuento de colonias, observándose que los 6 sujetos PAS $(+)$ tienen un recuento de UFC/ml. por encima de los PAS (-) y un solo sujeto PAS $(+)$ presentó mas de 400 $\mathrm{UFC} / \mathrm{ml}$, los otros cinco sujetos PAS $(+)$ presentaron menos de $400 \mathrm{UFC} / \mathrm{ml}$. Así mismo todos los sujetos PAS (-) presentaron menos de 400 UFC/ml. Realizando curvas de características operativas para el receptor (ROC) y pruebas de sensibilidad y especificidad, no se pudo determinar un punto de corte para el recuento de colonias por el cual sería probable el desarrollo de hifas y/o pseudohifas. El análisis de regresión logística mostró que la edad, sexo, prótesis, xerostomía, higiene oral, medicación y especie de cándida, no son factores de confusión para la relación de la variable hifas y/o pseudohifas de cándida.

\section{Discusión}

De los 120 sujetos adultos con mucosa oral clínicamente sana que participaron en el presente estudio, el género más frecuente fue el femenino con 56,67\% (68 sujetos), ( $>00,05)$. La presencia de hifas de cándida en un examen directo es indicativo de infección activa que se produce en el momento de la invasión de los tejidos (1), sin embargo en el presente estudio se encontró que seis (5\%) sujetos sanos presentaron hifas y/o pseudohifas de cándida. Estos resultados son semejantes a los de Arendorf y
Walker (10) quienes en 54 sujetos sin historia de candidiasis oral, encontraron hifas en tres de ellos (5,56\%). Rindum (1) también refiere haber encontrado estructuras de hifas o pseudohifas en 4 a 24\% de sujetos con mucosa oral sana quienes no eran fumadores.

Sturtevant (11) refiere que el reconocimiento de las células del huésped por la levadura patógena de la $C$. albicans es probablemente un paso esencial en la patogenia del desarrollo de la enfermedad. En el presente estudio ante la ausencia de evidencia clínica de candidiasis en estos sujetos y que han desarrollado hifas podría sugerir que existen otros factores del hongo capaces de generar la infección. Además, los factores del huésped como la IgA secretoria juega un rol importante en el control de la candidiasis oral al inhibir la adherencia de la cándida a las células del huésped (12). Aunque no se ha resuelto la relación entre la producción de hifas y la virulencia, la patogénesis de la infección podría estar determinada mas por el huésped que por el hongo ya que el organismo no infecta individuos saludables y sólo es capaz de invadir y dañar tejido cuando las defensas del huésped están local y sistémicamente comprometidas.

Aunque existe un acuerdo entre los investigadores con respecto a que las hifas de $C$. albicans están asociadas con su capacidad invasiva ya que muestran una mayor capacidad de adhesión y producción de proteinasas que ayudan a romper la integridad de la mucosa bucal, este incremento en su virulencia no es determinante por si solo (6). Entonces, ante la ausencia de signos clínicos en los sujetos del presente estudio podría sugerir que además de las características del hongo juega un rol muy importante las características del huésped para desarrollar la infección. Por otro lado, se ha demostrado "in vitro" que, temperaturas que oscilan entre $37^{\circ}$ C y $40^{\circ} \mathrm{C}$, pH entre 6,5 y 7,0; así como la presencia de una concentración inicial de blastosporas que no exceda de $10^{6}$ $\mathrm{UFC} / \mathrm{ml}$, son esenciales para el crecimiento de las hifas al cabo de varias horas y que a pH 2,6 las células adherentes de la $C$. albicans, son capaces de formar hifas (6).

En el presente estudio no se encontró relación entre la edad y la presencia de hifas y/o pseudohifas de cándida similar a lo encontrado por Arendorf y Walker (10). Aunque se encontró más mujeres portadoras de hifas de cándida, pero no se encontró diferencia estadísticamente significativa, estos resultados coinciden con los de Rindum (1), Arendorf y Walter (10), y Novorikawa et al. (13).

Por otro lado, diversos estudios refieren los distintos factores que condicionan la presencia y virulencia de la cándida $(3,4,6,9)$; así al evaluar el uso de prótesis dental, mostró tener una influencia altamente significativa en el desarrollo de hifas y/o pseudohifas $(\mathrm{p}=0,002)$ obteniéndose una probabilidad de 98,21\% de desarrollarse, similar al hallado por Novorikawa et al. (65,32\%) (13); pues las prótesis removibles y las superficies mucosas recubiertas por las bases protéticas son un reservorio especial para las levaduras orales (4). La mayor probabilidad de desarrollarse en portadores de prótesis dental se debe a una característica del hongo de adherirse no solo a las células epiteliales sino también a las superficies acrílicas de las prótesis (9); y aquellas superficies que se apoyan en la mucosa presentan una mayor concentración de cándida que aquellas que no presionan la mucosa $(10,14)$. Además, la 
estomatitis protésica, es la forma más común de la candidiasis oral y ha sido descrita hasta en $60 \%$ de las personas que son portadoras de prótesis dental (4).

Otro factor que mostró influencia altamente significativa en el desarrollo de hifas y/o pseudohifas fue la presencia de xerostomía $(\mathrm{p}=0,0002)$ obteniéndose una probabilidad de desarrollarse de 82,14\%. La xerostomía afecta de un modo fundamental a la colonización oral por cándida, tanto al disminuir la acción limpiadora mecánica, como al disminuir el pH y los productos antifúngicos, como la enzima lizosima.

En relación con la condición de higiene oral se encontró que de los seis sujetos con frotis positivo: uno con higiene oral buena, tres con higiene oral regular, uno con higiene oral mala, y en uno no fue aplicable ( $>>0,05)$. De otro lado, tampoco se encontró influencia significativa entre el uso de medicamentos y la presencia de hifas y/o pseudohifas de cándida; pues del total de sujetos, 14 de ellos refirieron usar medicamentos, pero sólo en 1 se encontró frotis (+). En 33 sujetos se aisló cándida, de ellos en 31 sujetos se aisló $C$. albicans (25,83\% del total), similar a lo encontrado por Novorikawa et al. (13). La especie C. albicans fue la que más se encontró en el estudio lo que nos confirmaría que es la especie más predominante en la microbiota oral.

Por otro lado, un análisis cuantitativo del número de UFC a partir de la saliva nos puede permitir diferenciar los portadores de los infectados, de este modo los sujetos con menos de 400 $\mathrm{UFC} / \mathrm{ml}$ serian portadores y los que presentarían mas de 400 UFC/ml estarían infectados (4). Sin embargo, en el presente estudio encontramos 5 sujetos que presentaron hifas y/o pseudohifas de cándida con cultivos menor a 400 UFC/ml sin lesiones clínicas de infección candidiásica y un solo sujeto con frotis $(+)$ con un cultivo mayor a 400 $\mathrm{UFC} / \mathrm{ml}$ (3300 UFC/ml) y sin lesiones clínicas evidentes de candidiasis; este sujeto presentaba xerostomía y usaba prótesis considerados factores predisponentes. Estos resultados nos podrían sugerir que la presencia de al- gunas hifas y/o pseudohifas de cándida pueden ser signos iniciales de una infección candidiásica y que si a esto se le agrega un cultivo mayor a 400 UFC/ $\mathrm{ml}$ podría aumentar el riesgo a hacer una lesión candidiásica.

Se sabe que la candidasis es la infección más oportunista del mundo, pero la simple presencia de C. albicans no representa una enfermedad; además, raramente amenaza la vida; sin embargo, la candidiasis bucal posee una cantidad sustancial de morbilidad que llama la atención, y su presencia puede servir de indicador clínico para otras enfermedades subyacentes significativas (5). En conclusión, la presencia de hifas y/o pseudohifas de cándida no es indicador de infección pero si podría ser un indicador de riesgo de desarrollar candidiasis. Los resultados del presente estudio demuestran que en algunos sujetos puede existir la presencia de hifas y/o pseudohifas de cándida en sujetos adultos con mucosa oral clínicamente saludable sin evidencia clínica de infección candidiásica.

\section{Agradecimiento}

A la Dra. Beatriz Bustamante Rufino, Jefe del Laboratorio de Micología del Instituto de Medicina Tropical “Alexander Von Humboldt” de la Universidad Peruana Cayetano Heredia y Carmen Castro Estupiñán, laboratorista clínica por su apoyo en el procesamiento de las muestras.

\section{Referencias bibliográficas}

1. Rindum J.L, Stenderup A, Holmstrup P. Identification of Candida albicans types related to healthy and pathological oral mucosa. J Oral Pathol Med 1994; 23:406-12.

2. Gonzáles MM, Mangiaterra M, Paniagua S. Aislamiento y tipificación de levaduras en relación con gingivitis marginal crónica y la respuesta inmune. [publicación periódica en línea] 2002. [24 pantallas]. Se encuentra disponible en: URL: http://www.unne.edu.ar/cyt/2002/ 03-Medicas/M-040.pdf.

3. Senet J.M. Risk factors and physiopathology of candidiasis.
Rev Iberoam Micol 1997; 14: 6-13.

4. Delgado W. y Aguirre J. M. Las Micosis orales en la era del sida. Rev. Iberoam Micol 1997; 14: 14-22.

5. Lynch, DP. Oral Candidiasis: History, classification, and clinical presentation. Oral Surg Oral Med Oral Pathol 1994; 78: 189-193.

6. Pardi G, Cardozo EI. Algunas consideraciones sobre Candida albicans como agente etiológico de candidiasis bucal. Acta Odontol Venez 2002; 40(1).

7. Navazesh M, Wood G, Brightman V. Relationship between salivary flow rates and Candida albicans counts. Oral Surg Oral Med Oral Pathol 1995; 80(3):284-288.

8. Barturen B, Quindos G, San Millán R et al. Distribución de los serotipos de Candida albicans en aislamientos clínicos de personas inmunocompetentes e inmunodeprimidas. Rev. Iberoam Micol 1996; 13: 10-13.

9. Poirier C, Chimenos E, Ferrer M, et al. Importancia de los factores predisponentes en la candidiasis bucal. [publicación periódica en línea] 1996. [24 pantallas]. Se encuentra disponible en URL:http:/ /www.uv.es/medicina-oral/ Revista_3/Poirier.htm

10. Arendorf TM, Walker DM. The prevalence and intraoral distribution of Candida albicans in man. Archs Oral Biol 1980; 25: 1-7.

11. Sturtevant J, Calderone R. Candida albicans adhesins: Biochemical aspects and virulence. Rev Iberoam Micol. 1997; 14: 90-97

12. Ponton J, Bikandi J, Moragues MD et al. Reactivity of Candida albicans Germ Tubes with salivary secretory Ig A. J Dent Res 1996; 75(12): 19791985

13. Novorikawa E, Sacsaquispe S, Varela L. Presencia de hifas y/o pseudohifas de cándida en mucosa oral clínicamente normal en adultos mayores. Diagnóstico 2005; 44(1): 23-26.

14. Biasoli M. y col. Efecto de la temperatura y el pH en la adherencia de Candida albicans in vitro. Rev Iberoam Micol 1999; 16: 46-49. 